\title{
An efficient and accurate MEMS accelerometer model with sense finger dynamics for applications in mixed-technology control loops
}

\author{
Chenxu Zhao, Leran Wang and Tom J. Kazmierski \\ School of Electronics and Computer Science \\ University of Southampton, UK \\ \{cz05r,lw04r,tjk\}@ecs.soton.ac.uk
}

\begin{abstract}
This contribution presents a novel MEMS accelerometer model implemented in VHDL-AMS. The model includes sense finger dynamics, which allow accurate performance prediction of a MEMS accelerometer in a mixed-technology control loop. A distributed mechanical sensing element model is developed and the effect of the sense finger dynamics is analyzed. The sense finger dynamics might cause a failure of the Sigma-Delta control loop which is captured by the proposed model but cannot be correctly modeled using the conventional approach.
\end{abstract}

\section{INTRODUCTION}

Micro-ElectroMechanical Systems (MEMS) require an integration of mechanical and electrical elements. These two disparate parts of a MEMS system have traditionally been designed separately using different methodologies and tools in different energy domains. Although several approaches have been proposed to mixed technology modeling of such systems [3], automated design methodologies for the whole integrated system supporting mixed physical domains are lagging behind. Recent AMS hardware description languages such as VHDL-AMS [1] are designed to support digital, ana$\log$ and mixed-signal systems but they cannot handle directly partial differential equations that are often needed to model distributed mechanical parts.

Due to the relatively high resolution and low temperature sensitivity, capacitive accelerometers are widely used in various industrial applications. Capacitive sensing of acceleration is also very well suited for applications that use closedloop control systems [4]. The MEMS capacitive accelerometer detects the mechanical displacement of the proof mass which is proportional to the input acceleration and translates it into an electrical signal, as the displacement of the proof mass changes the gap between the electrodes of capacitors, leading to a change in capacitance which can be measured easily. In order to improve the performance of the accelerometer in terms of bandwidth, dynamic range and linearity [2], and to convert analog acceleration signals directly to a digital form, electromechanical Sigma-Delta modulation (SDM) feedback control is usually applied. The output of such an accelerometer system is a digital pulse stream whose density represents the input acceleration.

The accelerometer proof mass is usually equipped with sense fingers placed between capacitor plates to increase the total capacitance variation when the proof mass moves (see Figure 1). The main drawback of this arragement is that finger resonance may affect the performance of the electromechanical Sigma-Delta modulator [5]. Sense fingers might bend seriously and oscillate at their resonant frequency leading to a failure of the oscillation of the Sigma-Delta control loop. However, the conventional approach normally applied in simulations of such systems, where a 2nd order lumped Mass-Damper-Spring equation is used to model the mechanical sensing element, cannot capture the effect of the sense finger dynamics. That means a failure of the Sigma-Delta control loop, when the fingers resonate, cannot be predicted correctly unless a more accurate modeling technique is used.

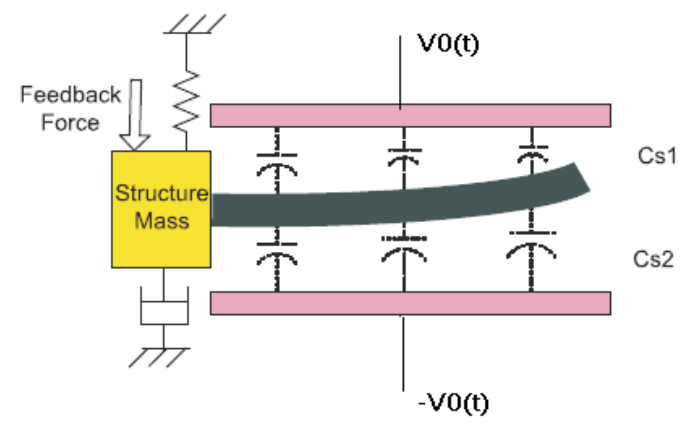

Figure 1: Distributed model for mechanical sensing element

This paper proposes a distributed approach where the sense fingers are modeled as cantilever beams whose motion can be described by Partial Differential Equations(PDEs). Since VHDL-AMS does not support PDEs, a Finite Difference Approximation (FDA) approach is applied to convert the PDE to a set of ODEs. Simulation results show that finger dynamics can be captured accurately using only a few discrete sections to approximate all the cantilever beams combined into a single equivalent sense finger.

\section{CONVENTIONAL MEMS DIGITAL AC- CELEROMETER MODELING}

Figure 1 show a simplified geometry of the accelerometer and the entire system in the closed-loop electromechanical Sigma-Delta modulator is shown in Figure 2. The structure mass in the mechanical sensing element is a Mass-DamperSpring system modeled by a 2 nd order differential equation: 


$$
f(t)=M \frac{d^{2} x}{d t^{2}}+D \frac{d x}{d t}+K x
$$

where $f(t)$ represents the input and feedback force, $M$ is the mass of the proof-mass, $x$ is the deflection of the proofmass, $D$ and $K$ are damping coefficient and spring constant respectively.

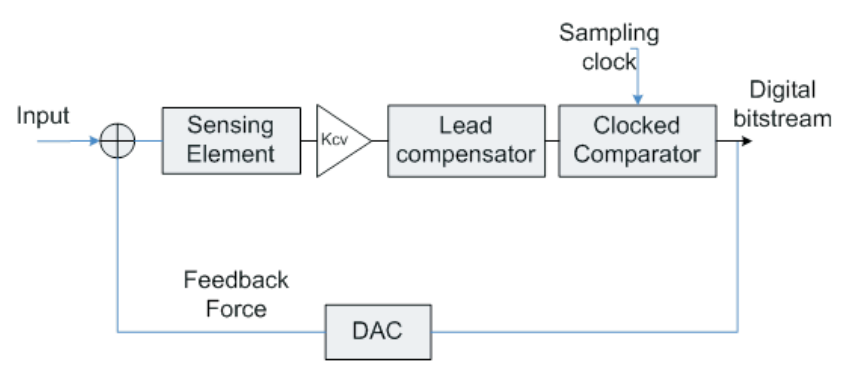

Figure 2: 2nd-order electromechanical Sigma-Delta accelerometer

The gain block $K_{c v}$ represents the signal pick-off from a differential change in capacitance to a voltage. The lead compensator is required to ensure the stability of the SigmaDelta feedback control loop. A clocked 1-bit quantizer is used for oversampling and generating pulse-density modulated digital signal. A pulse-density modulated feedback force is produced using the DAC. Figures 4 (a) and 5 (a) show simulation results of this model without accounting for finger dynamics. Figure 5 (a) indicates a correct operation of the system where the pulse density is inversely proportional to the input signal. In reality however, the sense element is vibrating at this input frequency thus rendering the feedback excitation ineffective, causing an incorrect output and a failure of the system [5]. This scenario cannot be reflected by the conventional model.

\section{SENSE FINGER DYNAMICS}

This section presents a more accurate approach to modeling the mechanical sensing element, which is distributed and can exhibit higher resonant modes. Such a model can be derived from the geometry of the sense electrode as illustrated in Figure 1. This is also a non-collocated system [5]. The feedback force is applied to the lumped mass(the root of the cantilever beam) and the electrostatic force is distributed along the length of the beam. $C_{s 1}$ and $C_{s 2}$ are the total distributed differential capacitances between the beam and the electrodes. $V_{0}(t)$ is the excitation carrier voltage.

The motion of the beam could be modeled by the following partial differential equation (PDE):

$$
\rho S \frac{\partial^{2} y(x, t)}{\partial t^{2}}+C_{D} I \frac{\partial^{5} y(x, t)}{\partial x^{4} \partial t}+E I \frac{\partial^{4} y(x, t)}{\partial x^{4}}=F_{e}(x, t)
$$

where $y(x, t)$ is a function of time and position that represents the deflection of the beam. $E, I, C_{D}, \rho, S$ are all physical properties of the beam: $\rho$ is the material density, $S$ is the cross sectional area $(W * T), W$ and $T$ are width and thickness of the beam, $E$ represents the Young's modulus which defines a material's shearing strength, $I$ is the second moment of area which could be calculated by $I=W T^{3} / 12$, $E I$ is usually regarded as the flexural stiffness, $C_{D}$ is the internal damping modulus, $F_{e}(x, t)$ is the distributed electrostatic force along the beam:

$$
F_{e}(x, t)=\frac{1}{2} \varepsilon A\left[\frac{V_{0}^{2}}{\left(d_{0}-y(x, t)\right)^{2}}-\frac{V_{0}^{2}}{\left(d_{0}+y(x, t)\right)^{2}}\right]
$$

where $\varepsilon$ is the permittivity, $\mathrm{A}$ is the area of the electrode, $d_{0}$ is the initial spacing between the beam and the electrode and $V_{0}$ is the amplitude of the applied excitation carrier voltage.

The boundary conditions of the cantilever beam are described by the following equations:

At $\operatorname{root}(\mathrm{x}=0)$

$$
\begin{aligned}
& y(0, t)=z(t) \\
& \frac{\partial y(0, t)}{\partial x}=0
\end{aligned}
$$

At free end $(\mathrm{x}=\mathrm{l})$

$$
\begin{aligned}
& \frac{\partial^{2} y(l, t)}{\partial x^{2}}=0 \\
& \frac{\partial^{3} y(l, t)}{\partial x^{3}}=0
\end{aligned}
$$

$z(t)$ is the deflection of the structure mass which could be modeled by a 2nd order differential equation as Equation 1:

$$
M \frac{d^{2} z(t)}{d t^{2}}+D \frac{d z(t)}{d t}+K z(t)=F_{\text {feedback }}+F_{\text {input }}
$$

In order to implement the above equation in VHDL-AMS, a Finite Difference Approximation (FDA) is applied to convert PDEs to a series of DAEs. Firstly, the beam is divided into $\mathrm{N}$ segments. 5 segments are chosen in this design as this number has been found adequate to reflect a failure of the system when the beam resonates. The deflection of the beam is discretized as:

$$
y_{n}(t)=y(n \triangle x, t) \quad n=0,1,2 \ldots N
$$

So the partial derivatives wrt position can be eliminated from Equation 3 and replaced with:

$$
\frac{\partial y_{n}(t)}{\partial x}=\frac{y_{n}(t)-y_{n-1}(t)}{\triangle x} \quad n=0,1,2 \ldots N
$$


Hence, the cantilever PDE (Equation 3 is converted to a set of Ordinary Differential Equations:

$$
\begin{array}{r}
\rho S \frac{d^{2} y_{n}}{d t^{2}}+\frac{C_{D} I}{(\triangle x)^{4}}\left(\frac{d y_{n+2}}{d t}-4 \frac{d y_{n+1}}{d t}+6 \frac{d y_{n}}{d t}\right. \\
\left.-4 \frac{d y_{n-1}}{d t}+\frac{d y_{n-2}}{d t}\right)+\frac{E I}{(\triangle x)^{4}}\left(y_{n+2}-4 y_{n+1}+6 y_{n}\right. \\
\left.-4 y_{n-1}+y_{n-2}\right)=\frac{f e_{n}(t)}{\triangle x} \quad(n=0,1,2 \ldots N)
\end{array}
$$

Equations for the border segments could be obtained from the boundary conditions. The complete, discretized distributed model consists of 6 DAEs shown in the following VHDL-AMS code.

library IEEE_PROPOSED;

Use IEEE_PROPOSED.ENERGY_SYSTEMS.all;

use IEEE_PROPOSED.ELECTRICAL_SYSTEMS.all;

use IEEE_PROPOSED.MECHANICAL_SYSTEMS.all;

library IEEE;

use IEEE.MATH_REAL.all;

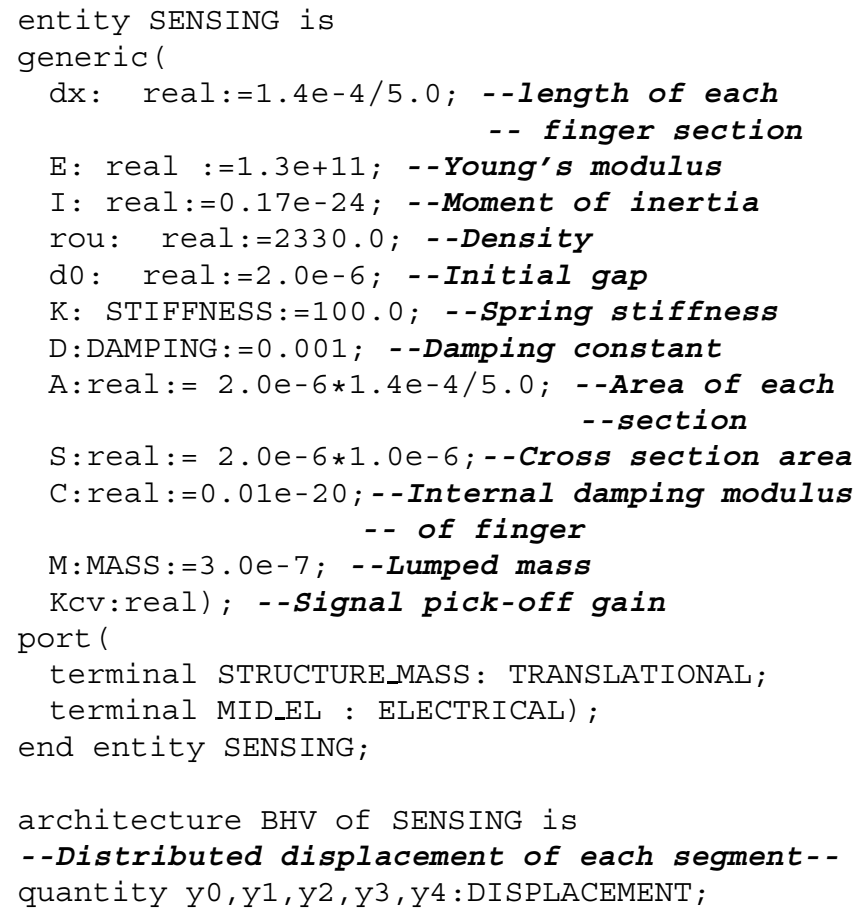

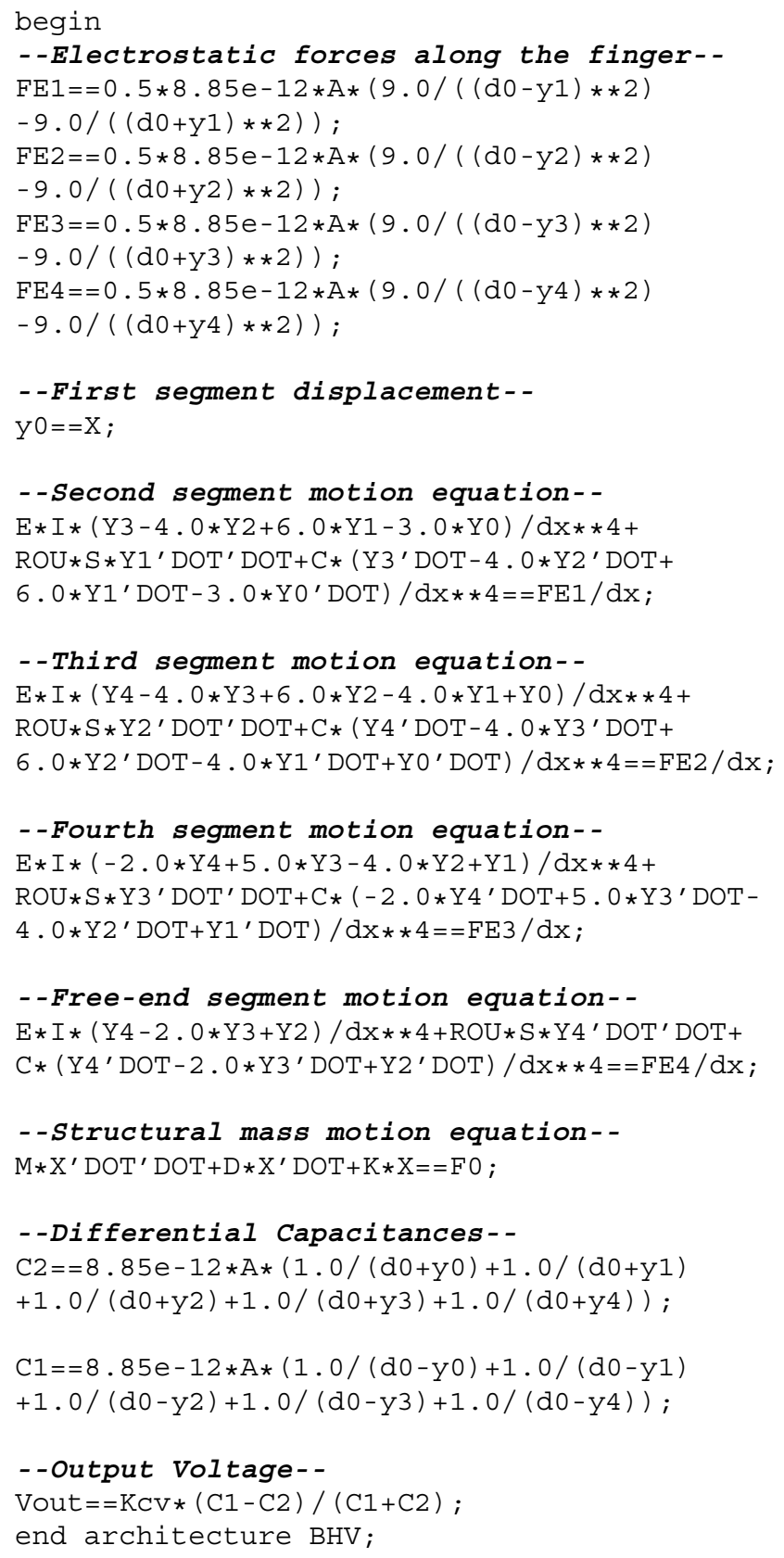

For this differential capacitive sensing element, the total distributed capacitance between the middle sense finger and electrodes is:

$$
\begin{aligned}
& C_{s 1}(t)=\frac{\varepsilon A}{n} \sum \frac{1}{d_{0}-y_{n}(t)} \\
& C_{s 2}(t)=\frac{\varepsilon A}{n} \sum \frac{1}{d_{0}+y_{n}(t)}
\end{aligned}
$$

where $\mathrm{n}$ is the number of the sections of the finger ( 5 sections in this model). The output voltage can be calculated as:

$$
V_{\text {out }}(t)=\frac{C_{s 1}-C_{s 2}}{C_{s 1}+C_{s 2}} K_{c v}
$$

The gain $K_{c v}$ represents the signal pick-off from a differential change in capacitance to a voltage. 
Like in the conventional model, the lowest resonant mode is caused by the dynamics of the structure mass, when the sense finger and lumped mass move together. The resonant frequency is approximately $w_{0}=\sqrt{K / M}$ where the $K$ is the suspension spring constant and $M$ is the total mass of the lumped mass and sense fingers. The higher resonant mode is related to the sense finger resonance, then the fingers bend significantly while the lumped mass has a small deflection. The resonant frequency could be calculated as that of the cantilever beam [5]:

$$
\omega_{i}=\alpha_{i}^{2} \frac{W}{L^{2}} \sqrt{\frac{E}{12 \rho}}
$$

The finger dimensions in this design are: $L=140 \mu \mathrm{m}, W=$ $2 \mu m, T=1 \mu m$. The first and second resonant frequencies are $60 \mathrm{KHz}$ and $385 \mathrm{KHz}$ respectively. Figure 3 shows the frequency response of this model which agrees well with the calculation results.

The sense finger resonance could affect the performance of the Sigma-Delta control loop as fingers might bend seriously and oscillate at their resonant frequency which causes a breakdown of the Sigma-Delta control. As shown in Figure 5 (b), fingers with the length $\mathrm{L}=200 \mu \mathrm{m}$ resonate and the Sigma-Delta control breaks down causing a fixed-density output waveform which does not reflect the input signal at all. The slight CPU time overhead related with the extra equations for finger dynamics is shown in the comparison between these two models in Table 1. SystemVision v.4 from Mentor Graphics was used to carry out the simulation experiments.

\begin{tabular}{c|l}
\hline MODEL & CPU TIME(SystemVision) \\
\hline Conventional Model & 9s $766 \mathrm{~ms}$ \\
\hline Distributed Model & 21s $812 \mathrm{~ms}$ \\
\hline
\end{tabular}

Table 1: CPU time comparison

\section{CONCLUSION}

An accurate MEMS accelerometer model with sense finger dynamics has been developed and implemented in VHDLAMS. The accelerometer operates in a high-sensitivity electrostatic Sigma-Delta control loop. Simulation results show that the proposed model correctly reflects the way in which finger dynamics affect the performance of the control loop. In contrast, the widely used conventional model of the accelerometer does not capture the well-known failure of the control loop when the system is excited with a frequency close to the sense finger resonance.

\section{REFERENCES}

[1] D.A.Teegarden, P.J.Ashendenm, and G.D.Peterson. The system designers guide to VHDL-AMS analogue, mixed-signal, and mixed-technology modelling. Morgan Kaufmann Publishers Inc, US, 10 Sep 2002.

[2] Y. Dong, M. Kraft, C. Gollasch, and W. Redman-White. A high-performance accelerometer with a fifth-order sigma-delta modulator. JOURNAL OF MICROMECHANICS AND MICROENGINEERING, 15:S22-S29, 2005.

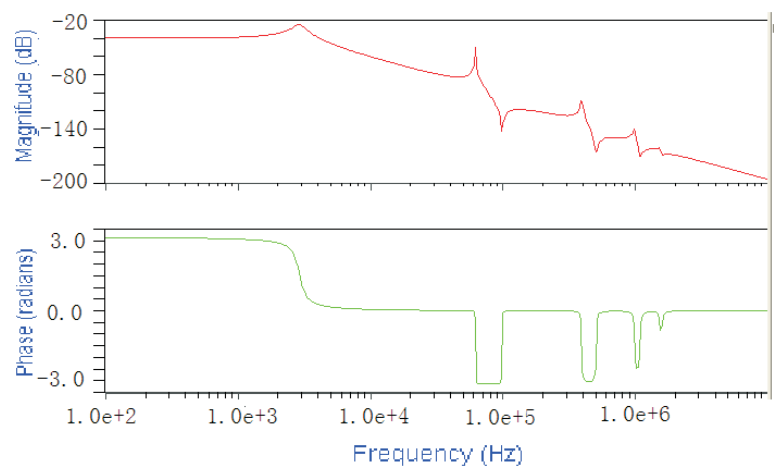

(a) Frequency response

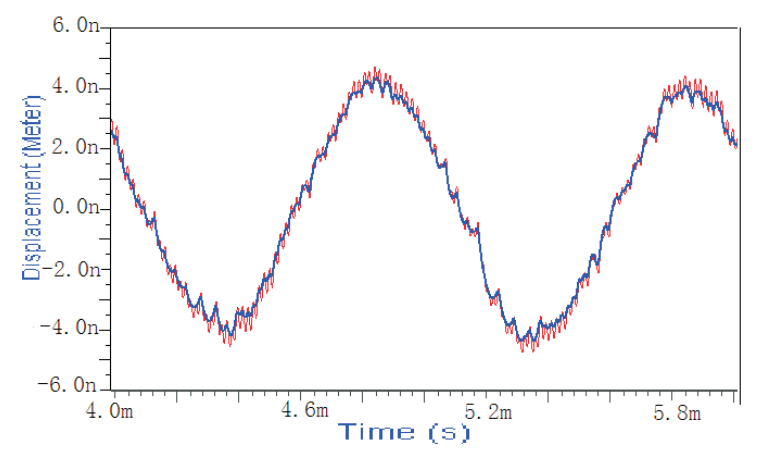

(b) Displacement of the lumped-mass (Blue) and average position of the beam (Red)

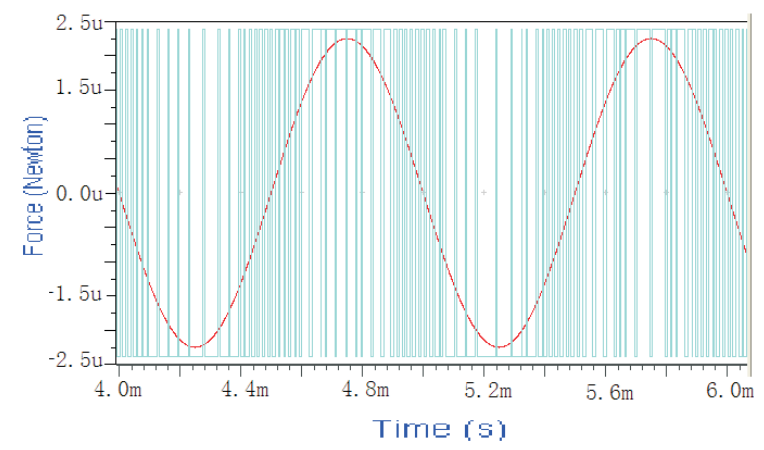

(c) Digital output bitstream

Figure 3: Simulation results for distributed model $(L=140 \mu m)$

[3] D. C. Hamill. Lumped equivalent circuits of magnetic components: The gyrator-capacitor approach. IEEE Trans. Power Electron, 8(2):97-103, Apr 1993.

[4] M. Kraft, C. Lewis, T. Hesketh, and S. Szymkowiak. A novel micromachined accelerometer capacitive interface. Sensors \& Actuators, A68/1-3:466-473, 1998.

[5] J. Seeger, X. Jiang, M. Kraft, and B. Boser. Sense finger dynamics in a $\sum \triangle$ force-feedback gyroscope. Tech. Digest of Solid State Sensor and Actuator Workshop, pages 296-299, Jun 2000. 


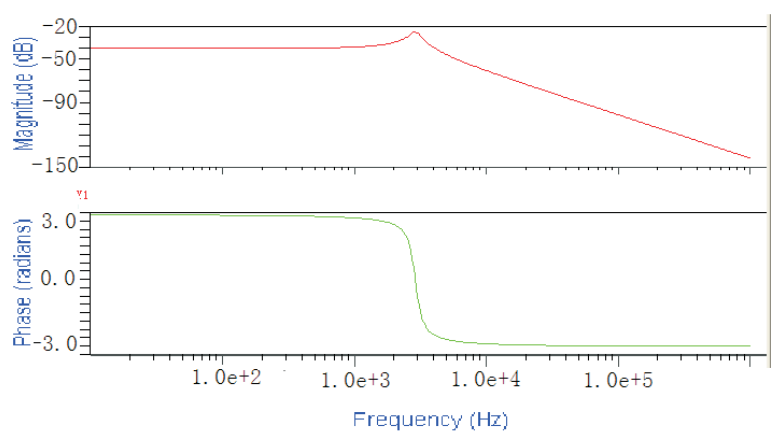

(a) Frequency response of the conventional lumped model

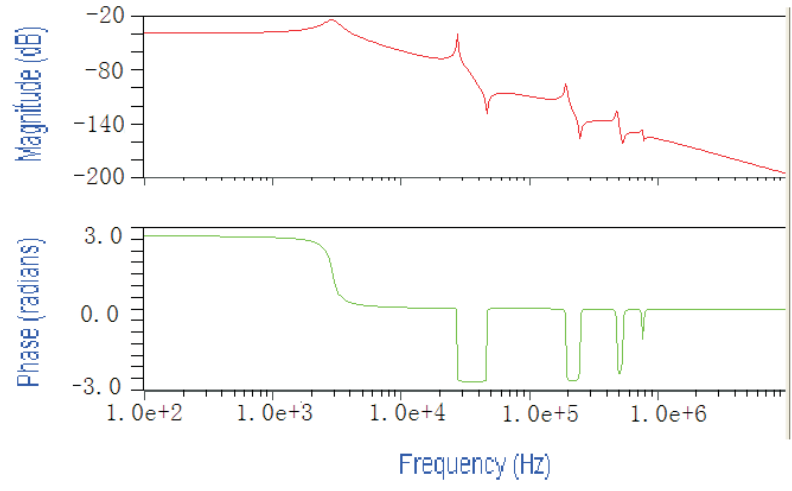

(b) Frequency response of the distributed model

Figure 4: Frequency response of the $\operatorname{models}(L=$ $200 \mu \mathrm{m})$

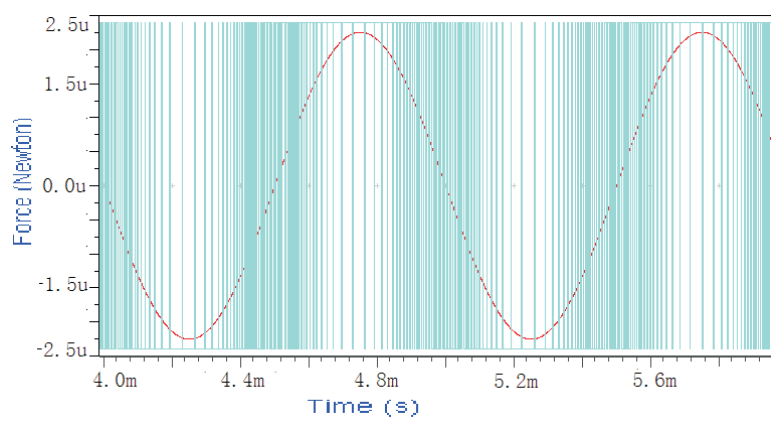

(a) Output bitstream of the conventional model. The Sigma-Delta control loop works while in reality the control breaks down. This behavior is incorrect.

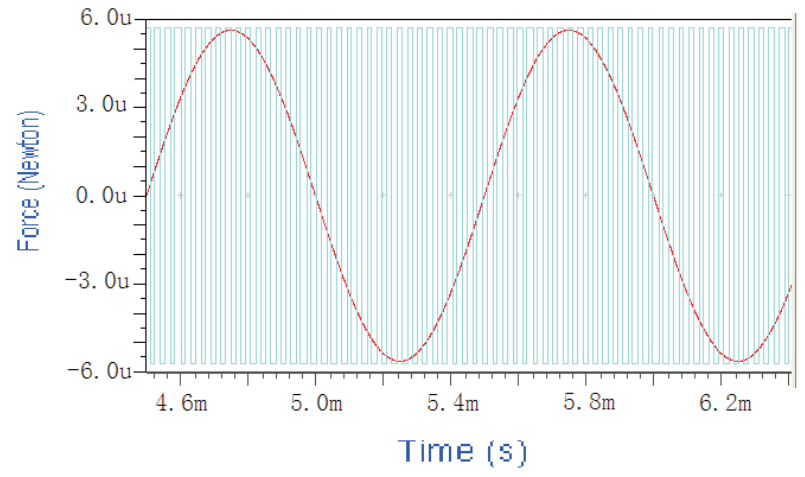

(b) Output bitstream of the proposed model showing the failure of the Sigma-Delta control loop at the finger resonant frequency. The proposed model correctly reflects this effect.

Figure 5: Simulation results showing that correct behavior is captured by the proposed model. 